\title{
Gestión de alto desempeño y su impacto en los resultados de la empresa: El caso de Uruguay y Argentina
}

\author{
John Miles ${ }^{1^{*}}$, Alberto González ${ }^{1}$, Natalia Mandirola
}

\begin{abstract}
Title: High performance management and their impact on business results: the case of Uruguay and Argentina
The main objective of the study is to identify which high-performance management practices have the greatest impact on business results. Using de Waal model, 56 executives from Uruguayan and 30 Argentine companies were interviewed, analyzing which practices prioritize, the progress in their implementation and their results. It is found that, regardless of the country, the characteristics of the company and of the executive, the practices related to continuous improvement and renewal have a greater impact on the essential results (profitability, income, growth). It is verified that, although the more a practice is prioritized, the better it is implemented, the results are significantly related to the progress in the implementation reaffirming the importance of the execution capacity.
\end{abstract}

Keywords: management practices; business results; high performance; Uruguay; Argentina.

Resumen: El objetivo principal del estudio es identificar qué prácticas de gestión de alto desempeño tienen mayor impacto en los resultados empresariales. Utilizando el modelo de Waal, se entrevistaron a 56 directivos de empresas uruguayas y 30 argentinas, se analizaron que prácticas priorizan, el avance en su implementación y sus resultados. La evidencia sugiere que, independientemente del país, de las características del directivo y de la empresa, las prácticas relacionadas con la mejora continua y renovación presentan un mayor impacto en los resultados esenciales (rentabilidad, facturación, crecimiento). Se verifica que, si bien cuanto más se prioriza una práctica, mejor se implementa, los resultados están relacionados significativamente al avance en la implementación reafirmando la importancia de la capacidad de ejecución

Palabras clave: prácticas de gestión; resultados empresariales; alto desempeño; Uruguay; Argentina.

Submitted: March $6^{\text {th }}, 2018 /$ Approved: May $18^{\text {th }}, 2018$

\section{Introducción}

Según el Banco Interamericano de Desarrollo (Grazzi \& Pietrobelli, 2016), en los últimos 15 años, América Latina presenta una disminución de la competitividad de sus empresas. Esta brecha ha venido aumentando con respecto al resto del mundo. A su vez, Bloom et al. (2010) presenta evidencias de que las empresas en países en desarrollo están usualmente mal administradas, lo cual substancialmente reduce su competitividad.

La competitividad de las organizaciones está explicada por factores del entorno, en general fuera de su control, y por factores internos relacionados con su gestión. Algunas investigaciones muestran que los factores internos tienen mayor incidencia (Algorta et al., 2012; Bloom et al., 2012a y 2017). Son estos los que explican por qué, en una misma industria -sometida a los mismos factores del entorno-, hay empresas que obtienen mejores resultados que otras.

La forma en que los directivos conducen su organización se manifiesta en prácticas de gestión. Se refieren a cómo se gestionan los procesos, la relación con los clientes y otras partes interesadas, la estrategia y su implementación, la fuerza laboral, entre otras.

Algunas prácticas de gestión están asociadas y conducen a mejores resultados y mayor competitividad (de Waal, 2012; Algorta et al.,
2012). Éstas, denominadas "prácticas de gestión de alto desempeño" son en principio todas buenas y relevantes, pero los directivos tienden a establecer prioridades entre las mismas, asociadas al perfil de la empresa, a la realidad del entorno o a la cultura gerencial, valores, experiencia y conocimiento del directivo. Esta priorización influye en los comportamientos del directivo y en el foco de la organización. Pero no alcanza con priorizar las prácticas adecuadas, es necesario implementarlas exitosamente para lograr resultados (de Waal, 2012; González et al., 2017; Bloom et al., 2017).

A fin de aportar conocimiento que ayude a mejorar la productividad de las organizaciones, en este trabajo se comprueba que existen un conjunto de prácticas que, al ser priorizadas por directivos de empresas uruguayas y argentinas, logran una mejor implementación e impactan positivamente en los resultados empresariales. Para ello, utilizando el modelo de prácticas de gestión de alto desempeño desarrollado por de Waal (2012), a través de entrevistas a una muestra intencional de directivos principales de 55 empresas uruguayas y 30 argentinas, que en los últimos años han tenido un buen desempeño, se releva las prácticas de gestión priorizadas, el avance logrado en su implementación y los resultados empresariales. Se testean tres hipótesis que relacionan la priorización de las prácticas, los avances en su implementación y los resultados empresariales, controlando con variables relacionadas al país, a la empresa y al directivo.

(1) Departamento de Ciencias de la Administración, Universidad Católica del Uruguay Montevideo, Uruguay

*Autor de correspondencia: jmiles@ucu.edu.uy 
Este trabajo está enmarcado en una investigación similar en curso en otros 4 países de América Latina. Las conclusiones son relevantes para las organizaciones de la región pues la mayor parte de las investigaciones de este tipo se realizan en economías avanzadas, mientras que las realizadas en países en desarrollo, en particular de la región, es algo limitada.

El trabajo se estructura en 6 apartados. En el siguiente presentamos la revisión de la literatura y el marco conceptual. En el tercero se desarrollan las hipótesis y en el cuarto se describe la muestra, el trabajo de campo y se indica la metodología utilizada para el contraste de las hipótesis. En el apartado cinco se describen los resultados y en el sexto se plantean las conclusiones, destacándose las implicancias para la gestión.

\section{Marco conceptual y revisión de literatura}

\section{La competitividad, la productividad y los resultados empresariales}

La competitividad de una empresa, y su eventual mejora, está determinada por factores relacionados al contexto y a la gestión. Al estudiar el impacto de estos factores en el desempeño de las empresas se observa que el análisis conjunto de factores asociados a la gestión y al entorno explica mejor la variación en los resultados de las empresas que cada uno por separado, confirmando su complementariedad (Hansen \&Wernerfelt, 1989; Mauri \& Michaels, 1998; Spanos \& Lioukas, 2001). Además, la evidencia empírica señala que la variación en los resultados empresariales se explica en mayor medida por el comportamiento de las variables asociadas con la gestión que por las asociadas con el entorno (Rumelt, 1991; Mauri \& Michaels, 1998; Miles, 2011; Algorta et al., 2012, Bloom et al., 2012a).

Algunas investigaciones confirman que en América Latina coexisten muchas empresas de baja productividad con algunas pocas que muestran altos niveles (Busso et al., 2013; Pagés, 2010). En la mayoría de los países de la región no solo sucede que diferentes sectores industriales muestran desempeños productivos disímiles, sino que este fenómeno está presente al interior de los sectores (Grazzi \& Pietrobelli, 2016). Las diferencias en la gestión explican, en gran parte, esta heterogeneidad.

En concreto, los estudios empíricos permiten afirmar que la mayor parte del desempeño de una empresa está determinado por cómo es liderada, cómo analiza el entorno y toma las decisiones, cómo planifica estratégica y operativamente, cómo define y elabora la propuesta de valor para sus clientes, cómo desarrolla e involucra al personal, cómo gestiona la información, los procesos y la tecnología, cómo innova su modelo de negocios y cómo se preocupa por lograr resultados balanceados para todas la partes interesadas. Ponemos especial énfasis en la palabra "cómo" ya que la clave del éxito empresarial no está tanto en las definiciones de "qué" hacer sino en "cómo" hacerlo, en la implementación. Lo que distingue a las organizaciones exitosas no es tanto "lo que hacen" sino "la forma cómo lo hacen" (Kaplan \& Norton, 2004; Algorta et. al, 2012; de Waal 2012, Bloom et al., 2012b; McKenzie \& Woodruff, 2017).
Sin embargo, la mayor parte de los esfuerzos teóricos y empíricos realizados para analizar las fuentes del retraso en la competitividad en la región, consideran el fenómeno a nivel agregado y con un mayor foco en los factores de entorno (Grazzi \& Pietrobelli, 2016). En particular en el Uruguay, como en otros países de la región, la importancia de los aspectos de gestión empresarial se ha subvalorado frente a los factores del contexto, que son lo que reciben mayor atención en las investigaciones, los medios de comunicación y en los foros empresariales. Esto puede conducir a una idea errónea de la capacidad que tiene la dirección de la empresa para mejorar su productividad, colocando la responsabilidad en factores del entorno, en general, fuera de su control, y limitando el desarrollo de acciones eficaces para aumentar su competitividad.

\section{Empresas de alto desempeño}

Aunque no existe una definición única de empresas de alto desempeño, varios autores coinciden en que son organizaciones con capacidad para satisfacer las necesidades y las expectativas de los clientes y otras partes interesadas, equilibradamente y a largo plazo, logrando mejores resultados financieros y no financieros que sus pares (Collins \& Porras, 1994; Mische, 2001; de Waal, 2012; Algorta et al., 2012).

Esta capacidad se manifiesta a través de prácticas de gestión incorporadas en rutinas y en la cultura empresarial, en modos de proceder. Hay prácticas que efectivamente conducen a productos y servicios de mejor calidad, a aportar mayor valor al cliente, a generar innovaciones y a la satisfacción de todas las partes interesadas, generando organizaciones más competitivas (Bloom et al., 2012a; de Waal, 2012; Algorta et al., 2012; Bigliardi \& Galati, 2014; González et al., 2017; Mckenzie \& Woodruff, 2017).

\section{Prácticas de gestión de alto desempeño}

En este trabajo utilizamos el modelo de de Waal para analizar la relación entre las prácticas de gestión y los resultados empresariales. André de Waal (2012) analizó 290 investigaciones y testeó posibles prácticas de gestión de alto desempeño en 1470 organizaciones de todo el mundo. Validó un modelo de 5 prácticas de gestión (agrupando 35 características), que tienen una correlación significativa con el alto desempeño y que parecerían ser genéricas para todo tipo de organización, industria y país. Son: 1) apertura y orientación a la acción 2) mejora continua y renovación, 3) alta calidad de los empleados, 4) compromiso a largo plazo 5) alta calidad del equipo directivo. De Waal confirmó que las empresas que prestan más atención a estas prácticas y que están más avanzados en su implementación obtienen mejores resultados que sus pares, en varias industria, sectores y países (de Waal et al., 2009; de Waal \& Frijns, 2011; de Waal \& Tan Akaraborworn, 2013; de Waal et al. 2015). En el Anexo 1 se describen las prácticas utilizadas en este trabajo.

La prioridad que los directivos asignan a las prácticas de gestión, y el avance en su implementación, puede depender de factores culturales y del entorno de negocios en el que se encuentra, de las características de la empresa y su negocio y de características personales de los directivos (Behrens, 2009; Hofstede et al., 2010; de Waal \& Chipeta, 2015; 
González et al. 2016). Sin embargo, estas diferencias no invalidan el modelo de prácticas de gestión de alto desempeño, que indica cuáles son las prácticas importantes para alcanzar resultados sostenibles, pero no la forma concreta en que las mismas se implementan. El "qué hacer" es genérico para las organizaciones que desean lograr el alto desempeño, mientras que el "cómo hacer" puede depender de factores culturales y del contexto. El modelo de de Waal indica qué prácticas de gestión una organización debe fortalecer para lograr el alto desempeño, pero no da una "receta" de cómo hacerlo ni establece un orden de prioridad (Richard et al., 2009; de Waal \& Chipeta, 2015). Además, como señalan Bloom et al. (2012a), las empresas multinacionales utilizan prácticas de gestión similares en muchos de los lugares donde actúan y, en la mayoría, obtienen resultados que son superiores a los de las firmas locales. Esto apoya la idea de que hay prácticas de gestión que, si son correctamente adaptadas e implementadas, generan resultados independientemente de los factores del entorno.

A su vez, Mckenzie y Woodruff (2017) y Bloom et al. (2017) encuentran que, si bien la buena gestión tiene un impacto positivo en los resultados de las organizaciones, son las prácticas más estructuradas las que presentan mayor efecto; sugiriendo que no todas las prácticas de gestión de alto desempeño tienen el mismo impacto en los resultados empresariales.

\section{Desarrollo de Hipótesis}

Las organizaciones son reflejo de las prioridades de los directivos. Aquellas prácticas de gestión que los directivos priorizan son las que reciben mayor atención y recursos y son las que logran avanzar más en su ejecución. Es importante determinar si esto es así, pues si lo es, los directivos que logren priorizar las prácticas de gestión de alto desempeño con mayor impacto en los resultados podrán efectivamente aumenta la productividad y competitividad de la organización.

H1: Las prácticas que son priorizadas por los directivos tienen mayor avance en su implementación.

No alcanza con priorizar las prácticas para generar resultados. Lo más importante es la capacidad de ejecución. Las organizaciones que son capaces de implementar las prácticas de gestión de alto desempeño obtienen mejores resultados empresariales.

$\mathrm{H} 2$ : Los resultados empresariales dependen positivamente del avance en la implementación de las prácticas de gestión de alto desempeño.

Existe un determinado paquete de prácticas de gestión de alto desempeño que pueden utilizar todas las organizaciones, independientemente de sus características y las características de los directivos, para mejorar su productividad.

H3 - Existen prácticas de gestión que tienen impacto positivo en los resultados empresariales en forma independiente de características de las empresas, del directivo y país.

\section{Metodología}

\subsection{Muestra}

Este estudio es de carácter exploratorio y el criterio de representatividad general no ha sido utilizado en la selección de la muestra, pues lo que importa en esta etapa es la identificación relaciones existentes entre las prácticas de gestión de alto desempeño y los resultados empresariales, dejando para una etapa posterior la posible generalización de resultados.

Partiendo de una lista amplia de empresas con buenos resultados de Uruguay y Argentina (Buenos Aires) elaborada en base a una consulta a expertos y referentes empresariales, se conformó una muestra con organizaciones que cumplían los criterios: a) reconocidas en su sector por su buen desempeño en los últimos años, b) que no hayan generado problemas importantes con alguna de sus partes interesadas, c) con actividad en contexto competitivo, y d) de diferentes tipos y sectores para conformar una muestra heterogénea (ver tabla 2).

Durante el 2015 y 2016 se entrevistaron a 56 directivos principales de organizaciones uruguayas y a 30 argentinas, con una antigüedad mínima de 5 años en la empresa y 3 en el cargo.

En las Tablas 1 y 2 se observa que las empresas y directivos estudiados de los dos países muestran algunas diferencias de perfil: mayor participación de empresa multinacionales en Argentina y mayor cantidad de empresas dirigidas por sus propietarios, de mayor edad y más antigüedad, en Uruguay. Por este motivo, en el análisis se utilizan variables de control relacionadas a las características de la empresa y relacionadas al directivo para evaluar su posible incidencia.

Tabla 1. Perfil de los directivos entrevistados.

\begin{tabular}{lcccc}
\hline \multirow{2}{*}{ Característica } & \multicolumn{2}{c}{ Argentina } & \multicolumn{2}{c}{ Uruguay } \\
\cline { 2 - 5 } & No. & $\%$ & No. & $\%$ \\
\hline Edad promedio (años) & 45 & - & 54 & - \\
\hline Propietario & 9 & $30 \%$ & 44 & $80 \%$ \\
\hline Contratado & 21 & $70 \%$ & 12 & $21 \%$ \\
\hline Antigüedad promedio (años) & 14 & - & 27 & - \\
\hline Hombres & 24 & $80 \%$ & 50 & $89 \%$ \\
\hline Mujeres & 6 & $20 \%$ & 6 & $11 \%$ \\
\hline
\end{tabular}

Tabla 2. Perfil de las empresas entrevistadas.

\begin{tabular}{llllll}
\hline \multirow{2}{*}{$\begin{array}{c}\text { Tipo de empresas } \\
\text { entrevistadas No. }\end{array}$} & \multicolumn{2}{c}{ Argentina } & \multicolumn{2}{c}{ Uruguay } \\
\cline { 2 - 6 } & Nacional & 12 & $40 \%$ & 38 & $69 \%$ \\
\cline { 2 - 6 } Origen & Multinacional & 18 & $60 \%$ & 17 & $31 \%$ \\
\hline \multirow{2}{*}{ Rubro } & Servicios & 11 & $37 \%$ & 25 & $45 \%$ \\
\cline { 2 - 6 } & Industria & 11 & $37 \%$ & 19 & $35 \%$ \\
\cline { 2 - 6 } & Otros & 8 & $27 \%$ & 11 & $20 \%$ \\
\hline
\end{tabular}




\subsection{Trabajo de Campo}

Como se mencionó anteriormente, el objetivo de este trabajo es evaluar las prácticas de gestión priorizadas por el directivo, la percepción que él tiene del avance en su implementación y la relación que ello tiene con los resultados de la empresa. En consecuencia, y sin desconocer los posibles sesgos de la metodología descripta, se entendió que no era pertinente realizar una triangulación de datos, entrevistando a otros actores de la organización, ya que la prioridad de las prácticas, la percepción del avance en su implementación y la percepción de los resultados empresariales son propias del directivo.

Durante la entrevista al directivo se le presentaban 5 tarjetas conteniendo, en cada una, el nombre y las características de una de las prácticas de gestión del modelo de de Waal (2012) (Anexo 1). De esta forma todos los entrevistados utilizaban exactamente las mismas definiciones. Primero se solicitaba que ordenara las prácticas de acuerdo a la prioridad que la misma tenía para su organización (en una escala de 1 a 5 , que en el análisis estadístico se lleva de 0 a 10 para homogeneizar los datos). Luego se le solicitaba el grado de avance en la implementación de cada una de las 5 prácticas, utilizando una escala de 0 (ningún avance) a 10 (mucho avance). Además, que mencionara ejemplos concretos de las prácticas para ayudar la interpretación de su respuesta.

Finalmente se solicitó información sobre un conjunto de resultados, financieros y no financieros. Considerando que muchos empresarios pueden no estar dispuestos a brindar información y datos contables sobre la rentabilidad, facturación, costos, etc., se utilizó un método indirecto. Varios trabajos empíricos han demostrado que las percepciones de los directivos sobre el desempeño de la organización: a) son un sustituto razonable de las medidas objetivas (Dess \& Robinson, 1984), y b) tienen una correlación significativa con medidas objetivas del desempeño (Venkatraman \& Ramaunujam, 1987; Hansen \& Wernerfelt, 1989; Spanos \& Lioukas, 2001). Atendiendo a lo anterior, se pregunta la opinión del directivo sobre la variación de los distintos resultados empresariales en los últimos tres años. Esta medición ha sido utilizada en otros estudios como: Spanos \& Lioukas (2001), Tippins \& Sohi (2003) y Miles (2011).

\subsection{Metodología para contrastar hipótesis}

Los resultados surgen de aplicar análisis de regresiones lineales múltiples y análisis factoriales a los valores asignados por los directivos a las priorizaciones, avances y resultados empresariales. Dada las características de la muestra (intencional y relativamente pequeña) las pruebas estadísticas fueron sometidas procedimientos de Bootstrap para confirmar su validez.

Para definir los resultados empresariales que se utilizan en los contrastes de las hipótesis, se realizó un análisis factorial para identificar posibles variables latentes que estructuren al conjunto de resultados relevados. Estas variables se toman como las variables dependientes en los análisis de regresiones.
Para contrastar la hipótesis 1, verificar si la priorización de las prácticas de gestión implica un mayor avance en su implementación, se realizaron regresiones lineales teniendo como variable dependiente el grado de avance en la implementación de la práctica y como independientes la priorización de la misma. En cada regresión solo se incorpora una de las prioridades como variable independiente debido a que todas las prioridades mantienen una alta colinealidad: siempre cuando una práctica recibe la máxima prioridad otra recibirá la mínima.

Se incluyen un conjunto de variables de control relacionadas con características de las empresas (país, tamaño, carácter exportador) y de los directivos entrevistados (si es propietario, edad y antigüedad en el puesto).

Para testar las hipótesis 2 y 3 se utilizan las dimensiones de resultados obtenidas en el análisis factorial como variables dependientes. En particular se realizaron dos análisis:

a. Correlaciones bivariadas (Pearson) entre los avances en la implementación de las prácticas y las dimensiones de resultados (Tabla 9).

b. Regresiones lineales múltiples, con las dimensiones de los resultados como variable dependiente y los avances en la implementación de prácticas como independiente. Además, se consideran las variables de control relacionadas con características de las empresas y de los directivos entrevistados (Tabla 10).

\section{Resultados}

La Tabla 3 muestra las prioridades y la Tabla 4 la distribución de los avances en la implementación de las prácticas de gestión. Tanto en el caso de prioridades y de avances las diferencias entre las medias de las prácticas son estadísticamente significativas.

Se observa que el Compromiso a Largo Plazo es la práctica más priorizada y con mayores avances en su implementación. En contrapartida Alta Calidad de los Empleados tiene menor priorización y menor avance. Y, de hecho, los directivos que priorizan Compromiso a Largo Plazo son los que menos priorizan Alta Calidad de los Empleados y viceversa.

Tabla 3. Priorización de las prácticas de gestión.

\begin{tabular}{lllll}
\hline \multirow{2}{*}{ Práctica } & \multicolumn{4}{c}{ Prioridad } \\
\cline { 2 - 5 } & Menor & Media & Mayor & Promedio \\
\hline 1. Apertura y orientación a la acción & 42 & 22 & 35 & 2,9 \\
2. Mejora continua y renovación & 48 & 21 & 31 & 2,7 \\
3. Alta calidad de los empleados & 60 & 12 & 28 & 2,5 \\
4. Compromiso a largo plazo & 22 & 12 & 66 & 3,8 \\
5. Alta calidad del equipo directivo & 35 & 18 & 47 & 3,2 \\
\hline
\end{tabular}

Nota: Menor: 1 o 2, Media: 3, Mayor: 4 o 5 
Tabla 4. Avances en la implementación de las prácticas de gestión.

\begin{tabular}{llllll}
\hline \multirow{2}{*}{ Práctica } & \multicolumn{5}{c}{ Avances en implementación } \\
\cline { 2 - 7 } & Bajo & Medio & Alto & Media & Mediana \\
\hline 1. Apertura y orientación a la acción & 1,2 & 19,8 & 79,0 & 7,5 & 8,0 \\
2. Mejora continua y renovación & 2,5 & 28,4 & 69,1 & 7,1 & 7,0 \\
3. Alta calidad de los empleados & 3,7 & 29,6 & 66,7 & 7,0 & 7,0 \\
4. Compromiso a largo plazo & 3,7 & 8,6 & 87,7 & 8,1 & 8,0 \\
5. Alta calidad del equipo directivo & 0,0 & 19,8 & 80,2 & 7,5 & 7,5 \\
\hline Nota: bajo:0 a 3, medio: 4 a 6, alto: 7 a 10
\end{tabular}

El nivel declarado de avance en la implementación es relativamente elevado, pero no extremo, mostrando una mirada realista por parte de los directivos.

Con respecto a los resultados, se indagaba cómo fue su variación en los últimos tres años, desde "disminuyeron mucho" a "aumentaron mucho". La Tabla 5 muestra los valores de los resultados consultados.

Tabla 5. Variación de los resultados en los tres últimos años.

\begin{tabular}{|c|c|c|c|c|}
\hline RESULTADOS & \multicolumn{4}{|c|}{$\%$ de empresas con ... } \\
\hline R1 - Satisfacción de los propietarios & 7,5 & 5,0 & 15,1 & 79,9 \\
\hline R2 - Satisfacción de los clientes & 6,9 & 6,1 & 20,7 & 73,2 \\
\hline R3 - Satisfacción de los empleados & 6,5 & 11,0 & 23,1 & 65.9 \\
\hline R5 - Acciones hacia la comunidad & 7,2 & 1,2 & 34,6 & 64,2 \\
\hline R6 - Acciones hacia el medio ambiente & 6,9 & 0,0 & 38,3 & 61,7 \\
\hline R7 - Posición en el mercado & 7,2 & 4,9 & 17,1 & 78,0 \\
\hline R8 - Volumen de clientes & 7,2 & 5,0 & 13,8 & 81,2 \\
\hline R11 - Facturación & 7,4 & 7,4 & 6,3 & 86,3 \\
\hline R12 - Rentabilidad & 6,1 & 21,5 & 16,5 & 62,0 \\
\hline
\end{tabular}

En todos los promedios predomina la declaración de que han avanzado, indicando que se trata de un conjunto de empresas con buenos resultados y que justifican su inclusión en el estudio. Se constata que existen diferencias importantes en la evaluación de los distintos resultados, mostrando que los directivos entrevistados contestan discriminando las variables.

Se realizó un análisis factorial para identificar posibles variables latentes que estructuren al conjunto de resultados. Los 12 resultados se terminan expresando en 3 dimensiones significativas. La Tabla 6 muestra el \% de varianza explicada por los factores y la Tabla 7 la composición de las diferentes dimensiones

Tabla 6. Porcentajes de varianza explicada.

\begin{tabular}{l|l|l|l}
\hline \multirow{2}{*}{ Dimensión } & \multicolumn{3}{l}{ Sumas de rotación de cargas al cuadrado } \\
\cline { 2 - 4 } & Total & \% de varianza & \% acumulado \\
\hline 1 & 3,05 & 25,44 & 25,44 \\
\hline 2 & 1,79 & 14,95 & 40,39 \\
\hline 3 & 1,56 & 13,04 & 53,43 \\
\hline Nota: $\mathrm{KMO}=0,77$ & \multicolumn{4}{l}{} \\
\hline
\end{tabular}

Tabla 7. Matriz de componentes rotados.

\begin{tabular}{|c|c|c|c|}
\hline \multirow{2}{*}{ Resultado } & \multicolumn{3}{|c|}{ Dimensión } \\
\hline & 1 & 2 & 3 \\
\hline R1 - Posición Mercado & 0,77 & $-0,02$ & 0,09 \\
\hline R2 - Facturación & 0,76 & 0,21 & 0,05 \\
\hline R3 - Rentabilidad & 0,73 & 0,30 & $-0,07$ \\
\hline R4 - Cantidad Personal & 0,61 & 0,25 & 0,08 \\
\hline R5 - Inversiones & 0,57 & 0,25 & 0,15 \\
\hline R6 - Satisfacción Clientes & 0,47 & 0,36 & $-0,22$ \\
\hline R7 - Volumen Clientes & 0,44 & 0,08 & 0,29 \\
\hline R8 - Satisfacción Empleados & 0,26 & 0,82 & 0,20 \\
\hline R9 - Satisfacción Propietarios & 0,21 & 0,77 & $-0,03$ \\
\hline R10 - Acciones Medio Ambiente & $-0,13$ & $-0,01$ & 0,76 \\
\hline R11 - Acciones hacia Comunidad & 0,12 & 0,34 & 0,70 \\
\hline R12 - Alianzas Estratégicas & 0,34 & $-0,16$ & 0,54 \\
\hline
\end{tabular}


La primera dimensión (25\% de la varianza), se puede interpretar como el factor de Resultados Esenciales, es decir aquellos que están directamente vinculados a la subsistencia de la empresa y que expresan su crecimiento (o decrecimiento). Estos resultados, en general, están medidos en estas organizaciones, por lo cual la percepción del directivo está muy cerca de la realidad. Crecimiento en facturación, inversión y personal son expresiones "duras" de una empresa en expansión.

La segunda dimensión ( $15 \%$ de la varianza), expresa los Resultados de Satisfacción Interna: de propietarios y de empleados. Es importante destacar que la percepción de la satisfacción interna aparece relativamente independiente de los Resultados Esenciales, mostrando que no alcanza tener resultados esenciales para lograr la satisfacción interna, se requiere gestionar otras variables vinculadas al clima organizacional. Es relevante la relación que aparece entre la satisfacción del propietario y la satisfacción de los empleados. Como el directivo (muchas veces propietario) es quien declara la satisfacción, esto implica que su satisfacción está vinculada a la percepción de la satisfacción del personal. Incluso se observa, que la satisfacción de los clientes también es relativamente importante en esta dimensión.

La tercera dimensión (13\% de la varianza), que denominamos "Resultados de Actividades en el Entorno", muestra la relación de la empresa con el entorno en línea con acciones que se asocian con la RSE. En este sentido un estudio previo sobre empresas uruguayas (Algorta et al., 2012) indicaba una independencia entre las acciones hacia al entorno y la comunidad y los resultados financieros. En el caso particular de las Alianzas Estratégicas, estas pueden tener un impacto muy fuerte en los resultados esenciales, lo cual resulta coherente con el peso que también tiene en el primer componente. De modo similar, las Acciones hacia la Comunidad, también se expresan con cierto peso en la dimensión de Satisfacción Interna, mostrando que pueden tener un impacto positivo en la satisfacción de los stakeholder internos.

Para comprobar la independencia de estas dimensiones de resultados respecto aspectos relacionados al tipo de empresa y las características del directivo se realizó un análisis de regresión tomando como variables explicativas las de control y como dependientes las dimensiones de resultados En ningún caso se encontraron relaciones significativas, confirmando la independencia del nivel y estructura de los resultados con la estructura de la muestra.

Los resultados del contraste de la hipótesis 1 se muestra en la tabla 8. Se observa que existe una relación positiva y significativa entre la priorización de una práctica de gestión y el avance en su implementación. Además, la incorporación de las variables de control no afecta esta relación positiva. Se ratifica la hipótesis de que las prácticas que son más priorizadas por los directivos tienen más avance en su implementación.

Esta conclusión no excluye la posibilidad -explícitamente indicada por algunos directivos- que se priorice una práctica porque la misma se encuentra en un nivel menos avanzado que las otras. La manera de evaluar el sentido de la causalidad: prioridad $\rightarrow$ avance o debilidad $\rightarrow$ prioridad implicaría estudios evolutivos que estuvieron fuera el objetivo de esta investigación.

Tabla 8. Resultados de las regresiones que asocian el avance en la implementación de las prácticas con las prioridades y variables de control

\begin{tabular}{|c|c|c|c|c|c|}
\hline & \multicolumn{5}{|c|}{ Avance en la implementación de la práctica priorizada } \\
\hline Constante & 8,144 & 5,488 & 6,817 & 7,916 & 7,466 \\
\hline \multicolumn{6}{|l|}{ Práctica priorizada } \\
\hline Alta calidad de los empleados & & & $0,374^{\star *}$ & & \\
\hline Compromiso a largo plazo & & & & $0,577^{\star *}$ & \\
\hline Alta calidad del equipo directivo & & & & & $0,358^{* *}$ \\
\hline \multicolumn{6}{|l|}{ Perfil de la empresa } \\
\hline País & 0,123 & 0,080 & 0,102 & 0,127 & 0,292 * \\
\hline \multicolumn{6}{|l|}{ Perfil del directivo } \\
\hline Propietario & 0,112 & 0,171 & 0,098 & $-0,087$ & 0,022 \\
\hline Edad & $-0,111$ & 0,006 & $-0,087$ & $-0,029$ & $-0,102$ \\
\hline Años en el puesto & 0,256 & 0,178 & $0,321 *$ & 0,059 & 0,171 \\
\hline Promedio (variable dependiente) & 7,45 & 7,12 & 7,04 & 8,10 & 7,54 \\
\hline
\end{tabular}


En las Tablas 9 y 10 se muestran los resultados de los análisis utilizados para contrastar la hipótesis 2 .

Tabla 9. Correlaciones entre resultados empresariales y avances en la implementación de las prácticas.

\begin{tabular}{llll}
\hline \multicolumn{4}{c}{ Resultados } \\
\hline PRÁCTICA & Esenciales & $\begin{array}{l}\text { Satisfacción } \\
\text { Interna }\end{array}$ & $\begin{array}{l}\text { Actividades } \\
\text { en el entorno }\end{array}$ \\
\hline Apertura y orientación a la acción & 0,174 & $-0,120$ & $-0,015$ \\
\hline Mejora continua y renovación & $0,237^{*}$ & $0,229^{*}$ & 0,123 \\
\hline Alta calidad de los empleados & $-0,069$ & 0,073 & 0,128 \\
\hline Compromiso a largo & $-0,043$ & 0,120 & 0,093 \\
\hline Alta calidad del equipo directivo & 0,096 & 0,168 & 0,021 \\
\hline Nota: Correlación de Pearson & $\begin{array}{l}* \text { La correlación es significativa en el nivel } \\
0,05(2 \text { colas) }\end{array}$ \\
\hline
\end{tabular}

La única práctica de gestión cuyo avance en la implementación muestra una correlación significativa con dos de las dimensiones de resultados es la Mejora continua y renovación (Tabla 9). Además, es la única cuyo avance en la implementación tiene un impacto positivo significativo sobre los resultados esenciales (Tabla 10). A su vez, tiene un impacto positivo sobre el resultado satisfacción interna mientras que la práctica apertura y orientación a la acción tiene un impacto negativo.

Ninguna de los avances en la implementación de las prácticas tiene un efecto significativo sobre los resultados de actividades en el entorno.

El impacto de Mejora continua y renovación sobre los resultados esenciales se hace significativo cuando se incluyen las variables de control (modelo 1.3, Tabla 10). Esto no le quita importancia a su efecto ya que efectivamente existe una correlación significativa entre esta práctica y los resultados esenciales (Tabla 9) que al realizar la regresión considerando solo los avances en las prácticas (modelo 1.1) puede ser en parte neutralizado por otras prácticas que tiene una correlación negativa, aunque no significativa, con los resultados esenciales (Tabla 9). Cuando se analiza el avance en la implementación de las prácticas y cada uno de los 12 resultados individualmente considerados se confirma que la práctica Mejora Continua y Renovación es la que tiene impacto en la mayor cantidad de resultados individuales, en particular: rentabilidad, facturación, posición en el mercado, así como en satisfacción de personal y de propietarios.

Podemos concluir que la correcta implementación de la práctica $\mathrm{Me-}$ jora Continua y Renovación es la que tiene mayor impacto en la organización en términos de Resultados Esenciales y Satisfacción Interna.

Por otro lado, se evaluó el efecto de la priorización sobre los resultados esenciales. Para ello se realizó un análisis de correlaciones bivariadas, ya que por la forma de relevar los datos de las prioridades las mismas presentan una alta colinealidad lo que inhabilita la utilización del análisis de regresiones. No se observa ninguna correlación significativa entre las prioridades de las prácticas de gestión y los resultados. Lo que implica que no alcanza con priorizar las prácticas para lograr impacto en los resultados esenciales.

En consecuencia, la hipótesis 2 se verifica parcialmente. Solo el avance en la implementación de la práctica Mejora continua y renovación tiene un impacto positivo y significativo sobre los resultados esenciales y de satisfacción interna; mientras que la Apertura y orientación a la acción muestra un impacto negativo sobre la satisfacción interna. Ninguna de los otros avances en implementación de prácticas muestra un efecto significativo sobre los resultados empresariales.

En la Tabla 10 se observa que al introducir las variables de control a través de una regresión múltiple ninguna de ellas aparece influenciando los resultados en forma significativa, por lo tanto, las tendencias mostradas son independientes del país, de los perfiles empresariales y de los directivos encuestados. Esto ratifica la $\mathrm{H} 3$ que sostiene que las prácticas tienen impacto en los resultados en forma independiente de características de las empresas, del directivo y país 
Tabla 10. Relación entre resultados empresariales y avances en la implementación de las prácticas

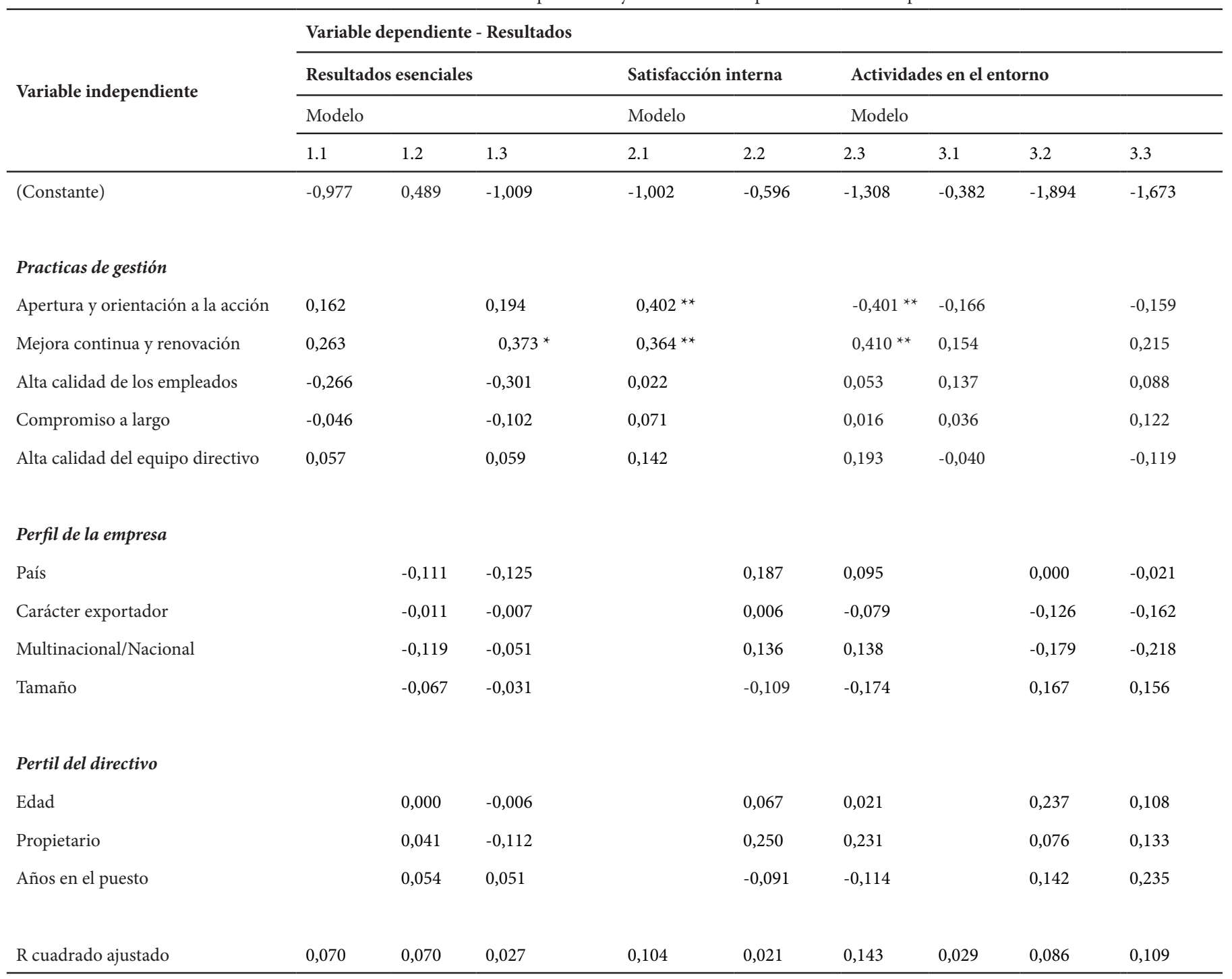

Nota: ${ }^{*}$ significativo al $5 \%$; ${ }^{* *}$ significativo al $1 \%$.

Los modelos 1.1, 1.2 y 1.3 presenta la regresión solamente con los avances en la implementación de las prácticas como variables independientes. En los modelos 2.1,2.2 y 2.3 las variables independientes son las de control. En los modelos 3.1, 3.2 y 3.3 la regresión incluye como variables independientes los avances en las prácticas y las variables de control

\section{Discusión y conclusiones}

Este trabajo pretende comprobar la existencia de una relación positiva entre un conjunto de prácticas de gestión de alto desempeño, priorizadas e implementadas por directivos de empresas uruguayas y argentinas, y los resultados empresariales, identificando aquellas que tienen mayor impacto. A través de entrevistas a una muestra intencional de directivos principales de 55 empresas uruguayas y 30 argentinas, que en los últimos años han tenido un buen desempeño, se relevaron las prácticas de gestión priorizadas, el avance logrado en su implementación y los resultados empresariales. Se testearon tres hipótesis que relacionan la priorización de las prácticas, los avances en su implementación y los resultados empresariales, controlando con variables relacionadas al país, a la empresa y al directivo.
Primeramente, se destaca que las empresas analizadas mejoraron sus resultados, tanto económico-financieros como de satisfacción de las otras partes interesadas y acciones en el entorno, pese a que los últimos tres años coinciden en ambos países con el trienio de menor crecimiento económico desde 2003-2004. También se verifica que están avanzadas en la implementación de las prácticas de gestión independientemente del país y de las características de las empresas y de los directivos. Esto permite considerarlas como casos de organizaciones de alto desempeño.

Se confirma la existencia de una relación positiva entre la práctica de gestión priorizada y los avances logrados en su implementación. Pero las prioridades no se expresan directamente en mejores resultados empresariales. Esto ratifica que no basta priorizar una práctica para lograr resultados, sino que se requiere una buena ejecución. 
Un constatación significativa es que la mejora en los Resultados Esenciales aparece asociada únicamente al avance en la implementación de la práctica Mejora Continua y Renovación, indicando que no todas las prácticas lograr el mismo impacto. Los aspectos claves contenidos en esta práctica: planificación estratégica, mejora continua de procesos, innovación, medición y seguimiento, y la comunicación de resultados claves constituyen una clara orientación de requisitos mínimos para el crecimiento de una empresa.

También se encuentra que la práctica Mejora Continua y Renovación tiene impacto positivo en la Satisfacción Interna (Propietarios y Empleados), posiblemente por estar asociada a mejores Resultados Esenciales en la organización. El impacto sobre los resultados de Actividades en el Entorno (Medio ambiente, Sociedad, Alianzas Estratégicas), no aparece relacionado a prioridades o avances ni a características de las empresas, directivos o país.

Salvo excepción, las variables de control asociadas tanto a la empresa como al directivo entrevistado no tienen impactos significativos en las prioridades ni en los avances de implementación de las prácticas de gestión. Es importante destacar la similitud encontrada entre las respuestas de los directivos argentinos y los uruguayos, teniendo en cuenta no sólo la diferencia de países, sino también el mayor tamaño y la mayor proporción de multinacionales en las empresas argentinas. Solo se encontraron diferencias en la prioridad Orientación a la Acción, siendo más fuerte en Argentina. Otra diferencia significativa fue en relación al rasgo si el directivo es propietario o no. Se encuentran una mayor prioridad de Compromiso a Largo Plazo, cuando el directivo es propietario, posiblemente asociado al compromiso personal del directivo con la empresa.

También se destaca que el país y las características de las empresas y de los directivos no aparecen asociadas a los Resultados Esenciales, ni a Satisfacción Interna, ni a Actividades en el Entorno. Esto confirma la existencia de prácticas de gestión que tienen impacto en los resultados empresariales independientemente de características de la empresa, del directivo o país. Para empresas que en los últimos años han operado en entornos económicos distintos, como el argentino y uruguayo, a mayor avance en la implementación de las prácticas de gestión ligadas a la Mejora Continua y Renovación mejores Resultados esenciales y resultados en la Satisfacción interna.

Finalmente, un hallazgo interesante es que los resultados aparecen estructurados en tres dimensiones, mostrando que el conjunto de Resultados esenciales (rentabilidad, facturación, participación de mercado, inversiones, satisfacción del cliente, crecimiento de personal) tienden a correr asociados. Pero el efecto en esta dimensión no garantiza la satisfacción de las partes interesadas internas (propietarios y personal) que pueden requerir políticas específicas y se genera el interrogante, de porqué la priorización en las prácticas de alta calidad de los empleados no aparece asociada a la dimensión de satisfacción.
Se pueden destacar las siguientes implicancias para la gestión:

Existe una relación positiva entre priorización de las prácticas de gestión y el avance en su implementación. Lo que prioriza el directivo es en lo que pone mayor atención y, en consecuencia, en lo que más se avanza. Por lo tanto, es importante conocer las prácticas de gestión que tienen mayor impacto en los resultados y aclarar las prioridades para alinear la acción de todos los integrantes de la organización. Los resultados están relacionados al avance en la implementación de las prácticas y no a la priorización de las mismas. O sea, para mejorar los resultados empresariales es necesario desarrollar la capacidad de ejecución, de implementar lo que se planifica y prioriza. Tal como señala Kaplan y Norton (2004), la mayoría de las organizaciones no fallan en la planificación sino en la implementación. Estas conclusiones son independientes del país y de las características del directivo y de la empresa. O sea, existen prácticas de gestión, que, si se priorizan y se logran implementar bien, tienen un impacto positivo en los resultados empresariales. Esto está en línea con las observaciones de Bloom et al. (2012b) que muestran que la empresa multinacionales, utilizando prácticas de gestión similares, con las necesarias adaptaciones a cada localidad, son las que tienen mayor productividad en todos los países donde actúan. Por lo tanto, los directivos pueden mejorar la competitividad de sus organizaciones implementando procesos de benchmarking que permitan adaptar e incorporar prácticas de gestión de empresas exitosas.

A su vez, el avance en la implementación de prácticas de gestión vinculadas a la Mejora Continua y Renovación son las que tienen mayor impacto en los Resultados esenciales de la organización y en la mayor cantidad de resultados particulares. Esto también está en línea con lo observado por Boom et al. (2017) en referencia a que organizaciones que utilizan prácticas de gestión más estructuradas tienen mayor productividad, rentabilidad, innovación y crecimiento. Estas constataciones ratifican la utilidad de los sistemas de gestión propuestos por la norma ISO 9001:2015 o los Modelos de Excelencia (EFQM o Malcolm Baldrige, por ejemplo) como instrumentos no solo para mejorar los procesos, productos y servicios de la organización, sino también sus resultados empresariales, financieros y no financieros.

Finalmente, el logro de Resultados esenciales (rentabilidad, facturación, participación de mercado, inversiones, satisfacción del cliente, crecimiento de personal) no es suficiente para generar buenos resultados en la satisfacción de los propietarios y del personal; es necesario tener políticas y desarrollar acciones específicas para ello.

Este estudio tiene la limitación de estar basado en una muestra intencional y relativamente pequeña, en consecuencia, sus conclusiones no son generalizables. El estudio se está expandiendo a otros países (Brasil, Colombia, Ecuador, Perú) con el objetivo indagar cuáles son las prácticas de gestión que, en general, pueden apoyar la mejora de la competitividad de empresas de América Latina y cuáles son más dependientes de las características de cada región. 


\section{Referencias bibliográficas}

Algorta, M., Auliso, R., González, A., Mandirola, N., Miles, J., Sorondo, A., y Zeballos, F. (2012). Prácticas de gestión que dan resultados. La experiencia de organizaciones de alto desempeño en el Uruguay. Montevideo, Uruguay: Grupo Magro.

Behrens, A. (2009). Culture and Management in the Americas. Stanford, USA: Stanford University Press.

Bigliardi, B. \& Galati, F. (2014). The Implementation of TQM in R\&D Environments. Journal of Technology Management \& Innovation, 9 (2), 157-171. doi:10.4067/s0718-27242014000200012

Bloom, N., Mahajan, A., McKenzie, D., \& Roberts, J. (2010). Why do firms in developing countries have low productivity? American Economic Review, 100(2), 619-623. doi:10.1257/aer.100.2.619

Bloom, N., Genakos, C., Sadun, R., \& Van Reenen. J. (2012a). Management Practices Across Firms and Countries. National Bureau of Economic Research, Working Paper No. 17850. doi:10.3386/ w17850

Bloom, N., Eifert, B., Mahajan, A., McKenzie, D., \& Roberts, J. (2012b). Does management matter? Evidence from India. The Quarterly Journal of Economics, 128(1), 1-51. doi:10.1093/qje/qjs044

Bloom, N., Brynjolfsson, E., Foster, L., Jarmin, R., Patnaik, M., Saporta-Eksten, I., \& Van Reenen, J. (2017). What Drives Differences in Management? National Bureau of Economic Research, Working Paper No. 23300. doi: $10.3386 /$ w23300

Collins, J., \& Porras, J. (1994). Built to last. Successful habits of visionary companies. New York, USA: Harper Business. doi:10.1002/ cir.3880060321

De Waal, A., Duong, H., \& Ton, V. (2009). High performance in Vietnam: the case of the Vietnamese banking industry. Journal of Transnational Management, 14(3), 179-201. doi:10.1080/15475770903120196

De Waal, A., \& Frijns, M. (2011). Longitudinal research into factors of high performance: the follow-up case of Nabil Bank. Measuring Business Excellence, 15(1), 4-19. doi:10.1108/13683041111113213

De Waal. A. (2012). What Makes a High Performance Organization: Five Factors of Competitive Advantage That Apply Worldwide. Hilversum, Netherland: Financial World Publishing.

De Waal, A., \& Tan Akaraborworn, C. (2013). Is the high-performance organization framework suitable for Thai organizations? Measuring Business Excellence, 17(4), 76-87. doi:10.1108/mbe-01-2013-0001

De Waal, A., \& Chipeta, K. (2015). Influence of culture on prioritysetting of high performance activities. Journal of Strategy and Management, 8(1), 64-86. doi:10.1108/jsma-05-2014-0034
Dess, G., \& Robinson, R. (1984). Measuring Organizational Performance in the Absence of Objective Measures: The Case of the Privately-held Firm and Conglomerate Business Unit. Strategic Management Journal, 5(3), 265-273. doi:10.1002/smj.4250050306

González, A., Mandirola, N. y Miles, J. (2016). Sustentabilidad: liderar organizaciones migrantes en el Uruguay - Aspectos asociados al alto desempeño empresarial. Journal of Technology Management \& Innovation, 11 (1), 55-64. doi: 10.4067/S0718-27242016000100008

González, A., Mandirola, N., Meléndez, H., y Miles, J. (Enero, 2017). Las prácticas de gestión de alto desempeño y los resultados empresariales. Un estudio comparativo entre empresas de Uruguay y Argentina. En: Memorias LI Asamblea Anual CLADEA 2016, La Innovación en las Escuelas de Negocio, CLADEA, Medellín. Recuperado de: http://201.234.64.127/Cladea2016/TRACKNo9/CLADEA_2016_paper_194.pdf

Grazzi, M. \& Pietrobelli C. (Ed.) (2016). Firm Innovation and Productivity in Latin America and the Caribbean. The engine of Economic Development. Washington, USA: Inter-American Development Bank. doi:10.1057/978-1-349-58151-1

Hansen, G., \& Wernerfelt, B. (1989). Determinants of firm performance: the relative importance of economic and organizational factors. Strategic Management Journal, 10(5), 399-411. doi:10.1002/ smj.4250100502

Hofstede, G., Hofstede, G.J., \& Minkov, M. (2010). Cultures and Organizations: Software of the Mind. New York, USA: McGraw-Hill.

Kaplan, R., y Norton D. (2004). Mapas Estratégicos. Convirtiendo los activos intangibles en resultados tangibles. Barcelona, España: Gestión 2000.

Mauri, A., \& Michaels, M. (1998). Firm and industry effects within Strategic Management: an empirical examination. Strategic Management Journal, 19(3), 211-219. doi:10.1002/(sici)10970266(199803)19:3\%3C211::aid-smj947\%3E3.0.co;2-t

McKenzie, D., \& Woodruff, C. (2017). Business Practices in Small Firms in Developing Countries. Management Science, 63(9), $2967-$ 2981. doi:10.1287/mnsc.2016.2492

Miles J. (2011). Análisis del Capital Intelectual de las Pequeñas y Medianas Empresas Uruguayas y su Impacto en los Resultados. Un estudio en las empresas desarrolladoras de software (Tesis Doctoral). Universidad de Deusto, San Sebastián, País Vasco.

Mische, M. (2001). Strategic renewal. Becoming a high-performance organization. Upper Saddle River, USA: Prentice Hall.

Pagés, C. (Ed.). (2010). La era de la productividad: cómo transformar las economías desde sus cimientos. Serie Desarrollo en las Américas. Washington, USA: Banco Interamericano de Desarrollo. Recuperado de: https://publications.iadb.org/bitstream/handle/11319/342/ DIA_2010_Spanish.pdf?sequence=1 
Richard, P., Devinney, T., Yip, G. \& Johnson, G. (2009). Measuring organizational performance as a dependent variable: towards methodological best practice. Journal of Management, 35(3), 718-804. doi:10.1177/0149206308330560

Rumelt, R. (1991). How much does industry matter? Strategic Management Journal, 12(3), 167-185. doi:10.1002/smj.4250120302

Spanos, Y., \& Lioukas, S. (2001). An Examination into the Causal Logic of Rent Generation: Contrasting Porter's Competitive Strategy Framework and the Resource Based Perspective. Strategic Management Journal, 22(10), 907-934. doi:10.1002/smj.174
Tippins, M., \& Sohi R. (2003). IT Competency and firm performance: is organizational learning a missing link? Strategic Management Journal, 24(8), 745-761. doi:10.1002/smj.337

Venkatraman, N., \& Ramanujam, V. (1987). Planning System Success: A Conceptualization and an Operational Model. Management Science, 33(6), 687-705. doi:10.1287/mnsc.33.6.687 


\section{Anexo I: Practicas}

Tarjetas para priorizar las prácticas. Cada tarjeta contiene el nombre de la práctica (en mayúscula) y una serie de características que la definen (en vignietas)

\section{APERTURA Y ORIENTACIÓN A LA ACCIÓN}

- Es flexible, estimula los cambios, se orienta al desempeño y la acción de todos

- Se involucra a todo el personal en los procesos

- Se les permite experimentar, arriesgarse y cometer errores

- Todos dedican tiempo a dialogar, compartir conocimiento y aprendizajes

\section{MEJORA CONTINUA Y RENOVACIÓN}

- Mantiene una estrategia única que la diferencia claramente del resto

- Mejora, simplifica y alinea sus procesos continuamente

- Innova en sus fortalezas, productos, servicios y procesos

- Mide y comunica aspectos clave de su rendimiento, financieros y no financieros

\section{ALTA CALIDAD DE LOS EMPLEADOS}

- Ensambla una fuerza de trabajo diversa y complementaria

- Inspira a sus integrantes a obtener resultados extraordinarios

- Les enseña a adaptarse a los cambios, ser flexibles, creativos y superar los obstáculos

- Les permite aprender de otros, mediante asociaciones con proveedores o clientes

\section{COMPROMISO A LARGO PLAZO}

- Procura satisfacer a sus clientes lo mejor posible

- Es un lugar de trabajo seguro para sus integrantes

- La dirección es duradera y genera nuevas direcciones desde la interna

- Mantiene relaciones duraderas con otras partes interesadas internas y externa

\section{ALTA CALIDAD DEL EQUIPO DIRECTIVO}

- Conduce con determinación y efectividad, se enfoca en los resultados a obtener

- Es un modelo a seguir por su ética, integridad, sinceridad y entusiasmo

- Mantiene relaciones de confianza con los empleados, valora su lealtad

- Los entrena, respeta sus competencias y actúa frente al bajo rendimiento 